\title{
Rursus
}

Russus

Poiétique, réception et réécriture des textes antiques

$8 \mid 2012$

Les épitomés scientifiques et historiques

\section{Calamità e prodigi nella storia di Roma repubblicana : la rilettura tardoantica di Orosio}

Calamités et prodiges dans l'histoire de la Rome républicaine : la relecture tardive d'Orose

Ida Gilda Mastrorosa

\section{OpenEdition}

\section{Journals}

Edizione digitale

URL: http://journals.openedition.org/rursus/1084

DOI: $10.4000 /$ rursus. 1084

ISSN: 1951-669X

\section{Editore}

Université Nice-Sophia Antipolis

Edizione cartacea

Data di pubblicazione: 14 dicembre 2012

Notizia bibliografica digitale

Ida Gilda Mastrorosa, «Calamità e prodigi nella storia di Roma repubblicana : la rilettura tardoantica di Orosio », Rursus [En ligne], 8 | 2012, mis en ligne le 11 décembre 2012, consulté le 20 avril 2019. URL : http://journals.openedition.org/rursus/1084; DOI : 10.4000/rursus.1084

Questo documento è stato generato automaticamente il 20 aprile 2019.

Rursus 


\section{Calamità e prodigi nella storia di Roma repubblicana : la rilettura tardoantica di Orosio}

Calamités et prodiges dans l'histoire de la Rome républicaine : la relecture tardive d'Orose

Ida Gilda Mastrorosa

\section{Introduzione: la storia come ricognizione di episodi funesti di epoca pagana a difesa dei cristiani}

1 Completate dal presbitero Orosio nei primi anni del v secolo d. C. ${ }^{1}$, le Historiae aduersus Paganos secondo quanto si legge nella Praefatio del I libro - come è noto - sarebbero state ispirate dall'invito di Agostino ad esprimersi contro l'atteggiamento dei pagani, pronti ad addebitare all'avvento del cristianesimo e all'abbandono degli antichi culti l'addensarsi di ogni genere di mali sull'umanità2, nonché più specificamente dal suo suggerimento di rileggere le opere anteriori per ricavarne dati su guerre funeste, epidemie, carestie, fenomeni naturali a carattere catastrofico :

Praeceperas ergo ut ex omnibus qui haberi ad praesens possunt historiarum atque annalium

fastis, quaecumque aut bellis grauia aut corrupta morbis aut fame tristia aut terrarum motibus terribilia aut inundationibus aquarum insolita aut eruptionibus ignium metuenda aut ictibus fulminum plagisque grandinum saeua uel etiam parricidiis flagitiisque misera per transacta retro saecula repperissem, ordinato breuiter uoluminis textu explicarem ${ }^{3}$.

Quale che sia il valore da attribuire alle dichiarazioni dello storico spagnolo e più in generale il significato da assegnare all'influenza esercitata su di lui dalla concezione storiografica del maestro ${ }^{4}$, non v'è dubbio che l'opera orosiana contenga significativi riferimenti a eventi calamitosi o eccezionali di vario tipo ${ }^{5}$. Tali riscontri, sovente coerenti per quanto concerne l'età repubblicana con le informazioni riportate in altre fonti fra cui i Libri ab urbe condita di Tito Livio ${ }^{6}$ e il Liber prodigiorum di Giulio Ossequente ${ }^{7}$, ove considerati alla luce delle testimonianze che danno parallela attestazione dei medesimi 
fatti, al di là della possibilità di individuare con chiarezza rapporti di dipendenza e mutuazione tra testi diversi ${ }^{8}$, aiutano comunque a mettere a fuoco le modalità di rilettura nella Tarda Antichità di episodi particolari della storia romana repubblicana e segnatamente a verificare quale reinterpretazione intendesse suggerirne un autore capace di declinare in modo peculiare prospettiva cristiana e istanze universalistiche.

\section{Concatenazione causale, contrazione dei tempi e accentuazione catastrofica nella riscrittura della storia dei secoli V-IV a. C.}

3 In tal senso, procedendo in ordine cronologico, un primo esempio di per sé significativo per notare innanzitutto l'attitudine di Orosio a reputare la soggezione dell'umanità a mali d'ogni tipo un dato costante e non circoscritto ad aree specifiche ${ }^{9}$ si ricava dal riferimento in un luogo del II libro ad un episodio pandemico accaduto a Roma sotto il consolato di Ebuzio e Servilio, vale a dire ascritto all'anno 463 a.C. In tale data, stando a quanto riportato nel passo ${ }^{10}$, si abbatté sull'urbe una pestilenza preannunciata da un improvviso bagliore di fuoco comparso nel cielo, interpretato come un prodigium che avrebbe anticipato il focolaio dell'epidemia poi divampata nella capitale, a causa della quale morirono i due consoli sopra ricordati e buona parte dell'esercito, furono inoltre contagiati molti nobili e soprattutto numerosi plebei, sicché si trattò di una nuova decimazione dopo quella accaduta quattro anni prima. Il confronto con le notizie attestate in relazione al medesimo evento nei Libri ab urbe condita ${ }^{11}$ lascia emergere la corrispondenza solo parziale dei dati. Il passo liviano, peraltro esemplificativo dell'attenzione riservata nell'opera ad eventi prodigiosi ${ }^{12}$ sulla scorta delle notizie registrate dalla tradizione annalistica ${ }^{13}$, non presenta alcun legame diretto fra l'ardere caelum (pur genericamente annoverato quale primo di una serie di portenta) e l'annus pestilens iniziato con l'ascesa dei due consoli e scandito dal dilagare del morbo con modalità di trasmissione sulle quali lo storico fornisce ipotesi distinte ${ }^{14}$, utili ad apprezzarne l'interesse per questioni di carattere medico ${ }^{15}$. Per converso, nel resoconto dei fatti dato da Orosio, non senza qualche riecheggiamento del racconto liviano, è significativa in primo luogo la scelta di classificare tecnicamente il fenomeno avvistato in cielo quale prodigium ${ }^{16} \mathrm{e}$ di correlarlo più specificamente alla grauis pestilentia verificatasi in seguito, con una connessione che emerge innanzitutto sul piano lessicale, grazie all'implicito parallelo tra l'improvviso ardere di quello e l'ignis morborum diffusosi nell'urbe. Caratterizzata da un certo tecnicismo connesso alla sfera medica ${ }^{17}$, la narrazione denota in particolare il ricorso a termini che insistono sull'idea del divampare per entrambi i fenomeni. Si tratta di un uso non inconsueto in relazione alla sfera epidemiologica oltre che in rapporto a specifici eventi di tipo astronomico ${ }^{18}$, tuttavia da valutarsi comunque in rapporto all'attitudine dello storico tardoantico a ricorrere ad immagini legate al fuoco ${ }^{19}$. Oltre al nesso istituito sul piano metaforico fra i due eventi, nella sua ricostruzione va tuttavia rilevata soprattutto la lettura del primo evento prodigioso quale premonizione del secondo (ut merito praecedente prodigio caelum ardere uisum sit, quando caput gentium tanto morborum igne flagrauit), in verità assente non soltanto nel racconto di Livio, bensì anche in quello di Dionigi d'Alicarnasso ${ }^{20}$, cui dobbiamo una puntuale descrizione dell'epidemia scoppiata nel 463 a. C. 
Quanto alle ragioni della "riscrittura" proposta nel passo delle Historiae aduersus Paganos, non si può escludere che grazie alla correlazione stabilita tra la pestilenza e il fenomeno celeste che la precedette Orosio mirasse a rimarcare in termini ancora più realistici il clima di terrore prodottosi in tempo di guerra, sia pure in una fase di intervallo delle operazioni militari, proprio a causa del verificarsi ravvicinato dei due fenomeni. La volontà di insistere su quest'ultimo aspetto, peraltro non estraneo alla mentalità romana ${ }^{21}$, potrebbe forse spiegare anche l'assenza nella versione orosiana di alcun riferimento alle misure che - stando a Livio - sarebbero state prese in seguito ai fenomeni sopra considerati, ovvero ai tre giorni di feriae indetti per placare gli animi, durante i quali i templi si affollarono di uomini e donne accorsi a invocare la pace degli dei ${ }^{22}$. Più specificamente, si può sospettare che, ove non scaturita dalla caduta della notizia nella fonte effettivamente usata da Orosio, la mancanza di alcun cenno sul punto specifico possa esser frutto della sua scelta di escludere dati utili a mostrare la cura rivolta dai pagani alla sfera religiosa, ovvero indicativi nel caso in questione di una pietas sensibile a recuperare la pax deorum. Alla luce di tale ipotesi, potrebbe dunque trattarsi di una soluzione narrativa ispirata dall'esigenza di passare sotto silenzio comportamenti attestanti il ruolo non marginale assegnato al cultus deorum dalla religiosità pagana, non trascurabile se consideriamo d'altro canto che in alcuni passi delle Historiae aduersus Paganos l'autore non rinuncia a rievocare pratiche pagane foriere di spunti adatti a consentirgli di manifestare con tono denigratorio le proprie riserve.

Eloquente in tal senso la ricostruzione dei fatti proposta in un luogo successivo del III libro $^{23}$. In tale contesto, dopo aver ricordato un episodio sismico occorso in alcune città dell'Acaia nel 373 a. C. ${ }^{24}$, accostandolo ad uno verificatosi ai suoi tempi, ovvero il terremoto di Costantinopoli del $396 \mathrm{~d}$. C., rievocato forse sulla scorta di informazioni tratte da Agostino ${ }^{25}$ e con il fine di sottolineare che nel caso più recente l'intervento di Dio, placato dalle preghiere dell'imperatore Arcadio, avrebbe evitato la rovina, lo storico menziona una pestilenza esplosa a Roma sotto il consolato di Lucio Genucio e Quinto Servilio, ovvero nel 365 a.C. Elaborato sulla base di notizie ancora una volta di derivazione incerta, comunque riscontrabili anche nel racconto di Livio $^{26}$, il passo documenta insieme all'interesse di Orosio per fenomeni sismici, analogamente rilevabile nei Libri ab urbe condita (TRAINA, $1996: 65, \mathrm{n} .35)^{27}$, anche il suo sforzo di annoverare con una certa puntualità, mista a toni di compassione enfatica ${ }^{28}$, probabili eziologie dell'episodio pandemico, chiamando in causa quali fattori scatenanti diverse tipologie meteorologiche (siccità invernale, improvvisi caldi primaverili, eccessive piogge estive) $\mathrm{e}$ l'intervento di venti miasmatici. Si tratta - a ben vedere - di particolari che, pur rimandando in modo indiretto a nozioni epidemiologiche attestate presso la tradizione antica $^{29}$, risultano al contempo superflui, data la scelta dell'autore di escludere fin da principio le diverse ipotesi poi menzionate (non ut adsolet...).

6 Cionondimeno la narrazione si segnala soprattutto per il riferimento nell'immediato seguito del discorso all'introduzione dei ludi scaenici appositamente suggerita nel 364 a. C. dai pontefici ai Romani per placare gli dei e porre fine all'epidemia ${ }^{30}:$ un dato destinato subito ad offrire ad Orosio uno spunto di valutazione negativa. Nonostante la motivazione addotta a premessa della notizia, ovvero il desiderio di non offrire ai possibili lettori di fede pagana l'occasione di rimproverargli omissioni volontarie ${ }^{31}$, il tono complessivo del racconto nonché la chiusa lasciano pochi dubbi sul fatto che con la menzione del rimedio espiatorio suggerito dai pontefici per ottenere un benevolo intervento degli dei contro la pestilentia, lo storico mirasse strumentalmente non già a restituire in modo completo $\mathrm{e}$ 
alieno da pregiudizi la verità dei fatti quanto piuttosto a procurarsi l'occasione di criticare le pratiche religiose pagane e segnatamente una misura a suo giudizio intesa all'apparenza a far cessare la pandemia ma in effetti foriera di mali spirituali (Ita pro depellenda temporali peste corporum arcessitus est perpetuus morbus animorum), dunque più dannosa del morbo a rimedio del quale fu escogitata (sequitur hanc miseram luem miserioremque eius expiationem ${ }^{32}$ ).

Ispirata sul punto specifico con ogni probabilità anche da analoghe posizioni di Agostino ${ }^{33}$ , la linea scelta da Orosio per la rievocazione dei fatti del 364-364 a. C. ove comparata con la soluzione dallo stesso adottata per la narrazione dell'episodio anteriore del $463 \mathrm{a}$. C. rende lecito ipotizzare che egli operasse una gestione oculata delle notizie ricavate dalle fonti, concedendosi ricostruzioni attente a registrare in modo differenziato particolari riguardanti la reazione dei pagani di fronte ad eventi calamitosi, vale a dire omettendo o integrando taluni dati a seconda che fossero utili a ricavarne argomento di riprovazione : quasi una sorta di "riscrittura" appositamente studiata, di cui reca traccia, del resto, proprio l'excusatio non petita posta a premessa del riferimento alla soluzione suggerita dai pontefici :

Conquererentur hoc, ut arbitror, loco obtrectatores temporis Christiani, si forte silentio praeterierim, quibus tunc caerimoniis Romani placauerint deos et sedauerint morbos ${ }^{34}$.

Che l'uso di dati su eventi a vario titolo funesti desunti dalla tradizione storiografica anteriore fosse concepito dallo storico come un'opportunità per spingersi a correlare situazioni in effetti diverse, probabilmente col proposito di farne le tessere di un mosaico recante la trama fitta e asfissiante di una storia dipanatasi come sequela ininterrotta di catastrofi e sventure, è testimoniato anche dal nesso stabilito nell'immediato seguito del passo tra la pestilenza sopra ricordata e un fatto eccezionale verificatosi l'anno successivo, ovvero un improvviso sprofondamento del terreno avvenuto evidentemente in prossimità di una voragine già formatasi in precedenza, che lasciando intravvedere quanto vi era sotto avrebbe suscitato un certo terrore e sarebbe stata usata per accogliere coloro che per volere degli dei fossero stati destinati ad esser sepolti da vivi ${ }^{35}$. Classificato subito come prodigium, tale cedimento del sottosuolo stando al racconto orosiano avrebbe di fatto preannunciato la disgrazia subito dopo occorsa a Marco Curzio : costui vi si sarebbe precipitato dentro, armato, insieme al suo cavallo, divenendo pasto di una terra dallo storico intesa metaforicamente come una bocca vorace e insaziabile, incapace di accontentarsi delle molte vittime dell'epidemia precedente e pronta ad inghiottire anche uomini vivi :

Satisfecit inprobis faucibus praecipitio sui M. Curtius, uir eques armatus, iniecitque crudeli terrae inopimam satietatem, cui parum esset, quod ex tanta pestilentia mortuos per sepulchra susciperet, nisi etiam uivos scissa sorberet ${ }^{36}$.

9 Accanto all'enfatizzazione giocata sul piano lessicale, nel passo va notato il riadattamento strumentale delle notizie attestate dalla tradizione e segnatamente da Livio, presso cui tuttavia si registra l'esistenza di una duplice versione dei fatti. Stando alla prima ${ }^{37}$, durante lo scontro consumatosi ai primordi dell'urbe fra Sabini e Romani, il capo dei primi Mettius Curtius sarebbe finito in una palude che, dopo il conseguimento dell'accordo fra le due comunità, sarebbe stata denominata Lacus Curtius in memoria dell'episodio e del suo protagonista comunque riuscito a sottrarvisi. Quanto alla seconda ${ }^{38}$, riportata in un passo successivo e privilegiata sia pur con cautela dallo storico patavino, ne ricaviamo che nell'anno del consolato di Quinto Servilio Ahala e Lucio Genucio, dunque nel 362 a. C., in seguito ad un terremoto o a qualche evento analogo nel centro del foro si sarebbe aperto 
un baratro che si cercò invano di colmare gettandovi della terra finché, consultati gli dei, si seppe che occorreva cercare quale fosse il principale fondamento della potenza romana (quo plurimum populus Romanus posset) e poi consacrarlo a quel luogo così da consentire che essa durasse in eterno. Alla luce di tale richiesta, Marco Curzio, dopo aver fatto rilevare che non poteva che trattarsi delle armi e del coraggio in campo bellico, secondo il racconto sarebbe salito sul suo cavallo e si sarebbe sacrificato gettandosi nella voragine che, colmata di numerose offerte votive, avrebbe poi preso il nome del protagonista di quel gesto eroico. A fronte delle due versioni conservate da Livio, è facile notare che Orosio, pur optando per la seconda, come lascia intendere in primo luogo l'assegnazione del fatto al al periodo successivo agli eventi del 365-364 a.C. e non all'età romulea, ne altera in qualche modo i dettagli, trasformando un atto di eroismo volontario in un esempio indicativo del fitto succedersi di eventi catastrofici nella storia di Roma repubblicana, perfino preannunciato da uno di essi, sicché la sua ricostruzione finisce con l'attestare il ricordo di uno sprofondamento del sottosuolo verificatosi in veste di presagio della disgrazia poi accaduta a Mezio Curzio, dunque un cedimento del terreno non scaturito da un evento di carattere sismico come si evince dal passo liviano (seu motu terrae seu qua ui alia...) bensì dopo un episodio di carattere epidemico con il quale concorre a rafforzare l'immagine di una terra crudelis protesa ad inghiottire senza sosta l'umanità.

10 L'ipotesi di una reinterpretazione ispirata dalla tendenza ad accentuare la dimensione catastrofica della storia anteriore all'avvento del cristianesimo può essere formulata anche per un altro passo del III libro in cui Orosio attesta due fenomeni meteorologici di natura particolare, ovvero il prolungarsi delle ore notturne e la caduta di chicchi di grandine di dimensioni eccezionali, verificatisi nel 348 a. C. in concomitanza con la stipulazione del I trattato romano-cartaginese ${ }^{39}$. Come è stato suggerito (LIPPOLD, 1976, I : $414)^{40}$, la notizia può essere accostata alla testimonianza di un presagio analogo menzionato per una data posteriore da Livio $^{41}$ secondo cui nel $344 \mathrm{a}$.C., dopo la consacrazione del Tempio di Giunone Moneta, avrebbe avuto luogo un'improvvisa caduta di pietre dal cielo che avrebbe provocato un religioso timore e indotto alla consultazione dei Libri sacri nonché alla conseguente decisione del senato di nominare un dittatore per indire feste solenni. Al di là della possibilità di riconoscere la matrice dell'informazione relativa ai due eventi naturali straordinari, va tuttavia rilevato che la particolare associazione stabilita tra questi e il primo accordo siglato dai Romani con la grande potenza rivale costituisce comunque un'innovazione di Orosio utile a percepire il suo strumentale ricorso ad attestazioni di fatti singolari : oltre ad implicare, come sembra probabile, un'anticipazione cronologica del caso ricordato nei Libri ab urbe condita, richiamando comunque l'attenzione del lettore sul carattere cruciale di un episodio storico destinato più avanti a dar materia come casus belli, egli sembra innanzitutto interessato a suggerire che anche in quella precisa circostanza la natura avesse lasciato presagire attraverso segni particolari quali sciagure avrebbero fatto seguito ad un evento così rilevante quale il primo foedus raggiunto fra Romani e Cartaginesi ${ }^{42}$.

11 Esplicitata nel seguito del discorso sul piano metaforico grazie all'immagine della "grandine di mali » sopraggiunta dopo l'arrivo di questi ultimi nella penisola ${ }^{43}$, la relazione istituita nel passo fra fenomeni straordinari e un episodio considerato epocale per le sue ricadute, quali che siano i possibili spunti e paralleli identificabili in altri contesti letterari, offre in definitiva un ulteriore esempio dell'inclinazione di Orosio a ricercare in accadimenti singolari i segni premonitori di sventure di ben altra portata. $\mathrm{Si}$ tratta di un'attitudine in verità testimoniata nel passo delle Historiae aduersus Paganos 
anche dalla scelta di menzionare in concomitanza la nascita di Alessandro Magno ${ }^{44}$, interpretata per traslato quale bufera rovinosa abbattutasi sull'Oriente, entro una prospettiva che dunque si amplia progressivamente fino a proiettare in chiave universale la soggezione dell'uomo a sciagure d'ogni tipo.

\section{II racconto di eventi luttuosi dei secoli III-II a. C.: un'occasione di critica di culti e credenze pagane e di censura del bellicismo iniquo dei Romani e dei loro nemici}

Oltre il passo appena considerato, probabilmente ispirato a livello più generale dalla volontà di insistere sul significato funesto implicito nel primo incontro di Roma con la potenza cartaginese $\mathrm{e}^{45}$, ulteriori attestazioni da Orosio fornite a proposito di calamità verificatesi in età mediorepubblicana, in particolare nei secoli III e II a. C., paiono ubbidire prevalentemente alla finalità di porre in cattiva luce i culti pagani. In tal senso va considerata la "riscrittura" della vittoria riportata da Lucio Papirio Cursore nel 293 a. C. contro i Sanniti proposta in un altro passo del III libro dove lo storico sostiene che essa sarebbe stata ottenuta a dispetto dei divieti formulati dagli augures pullarii ${ }^{46}$, avanzando una lettura dei fatti discorde rispetto alla tradizione ${ }^{47}$ ma soprattutto utile a consentirgli di ridicolizzare e guardare con scetticismo alle pratiche di sacerdozi inadeguati a predire gli eventi in modo corretto, come si evince da una valutazione successiva che d'altro canto documenta ancora una volta l'inclinazione dello storico a stabilire connessioni strumentali tra episodi diversi, in particolare attraverso la menzione dell'epidemia che comunque fece seguito alla battaglia condotta con successo dal console: Sed hanc quoque istius uere laudandam uictoriam, quam uani auspices impedire non potuerunt, oborti subito corrupere morbi. Efficace per ribadire l'idea del perenne soggiacere dell'umanità alle sventure, il nesso evidenziato tra i due episodi, non percepibile ad esempio nella tradizione accolta da Livio secondo cui sarebbe intercorso fra essi un anno, offre in effetti ad Orosio l'opportunità di proseguire in modo più esplicito la sua demolizione dei culti pagani, richiamando l'attenzione sul fatto che a causa del dilagare di una pestilentia di proporzioni eclatanti posteriore al successo di Papirio si ritenne necessario consultare i libri Sibillini e sulla base delle indicazioni ricevutene fu permesso l'arrivo a Roma dell'orrendo serpente (horrendumque... colubrum) di Epidauro con la pietra di Esculapio ${ }^{48}$.

13 È nella menzione critica di tale culto, giudicato inutile alla luce delle esperienze passate e inadeguato in rapporto al riaffacciarsi del problema dopo l'episodio considerato (quasi uero pestilentia aut ante sedata non sit aut post orta non fuerit) che si può cogliere ancora una volta la tendenza dello storico ad ordinare in modo strumentale alcuni dati recepiti dalla tradizione ${ }^{49}$, vale a dire per ottenerne occasioni di denigrazione dei riti pagani che d'altro canto lungi dall'apparire gratuite risultano invece tanto più legittime in quanto inserite entro un'originale ricostruzione dei fatti appositamente articolata in modo da far risaltare contrastivamente l'ineluttabile soggezione dell'umanità a sventure d'ogni tipo. Nonostante la corrispondenza con il giudizio critico riservato al culto di Esculapio da Agostino $^{50}$, la particolare correlazione da Orosio suggerita tra l'episodio che portò all'ingresso di quello e il caso di Papirio evidenzia infatti una peculiare attitudine a contrarre la successione temporale dei fatti e ad alterare connessioni causali tra eventi 
diversi così da far emergere in chiave storiografica l'inefficacia di credenze respinte innanzitutto sulla base di posizioni cristiane.

La tendenza a coordinare eventi attestati separatamente nelle fonti connota anche la registrazione di altri fenomeni straordinari verificatisi nei decenni successivi del III sec. a. C. Significativi al riguardo alcuni passi del IV libro delle Historiae aduersus Paganos riguardanti la fase della guerra di Roma contro Taranto ${ }^{51}$ e segnatamente gli scontri con Pirro. In tale contesto, data notizia di una tempesta avvenuta prima della battaglia di Ascoli del 279 a. C., accompagnata da tuoni fragorosi e perfino da fulmini abbattutisi sui portatori di foraggio al seguito delle truppe nonché sulle bestie ${ }^{52}$, Orosio indugia su una sequela di epidemie esplose durante gli intervalli dalle operazioni militari, rievocandole metaforicamente e leggendovi il segno dell'ira divina : Sed Romanorum miseria nullis cessat indutiis; consumitur morborum malis intercapedo bellorum et, cum foris cessatur proelio, agitur introrsum ira de caelo ${ }^{53}$. Entro tale cornice la sua attenzione si appunta in particolare su una pestilenza sviluppatasi sotto il consolato di Fabio Gurgite e Gaio Genucio Clepsina, dunque nel 276 a. C., abbattutasi soprattutto sulle donne e le femmine degli animali procurando la morte dei feti nel grembo delle madri o parti immaturi con i quali si davano alla luce degli aborti ${ }^{54}$. Benché la mancanza dei libri liviani relativi al periodo in questione non consenta di valutare la portata della "riscrittura" orosiana né sia possibile stabilire con certezza un'eventuale derivazione da materiali epitomati probabilmente ascrivibile - come è stato suggerito ${ }^{55}$ - anche ad un passo dall'analogo contenuto del De ciuitate Dei di Agostino, rimane significativo ai nostri fini che Orosio si avvalga di una tipologia di notizie attestata e interpretata in chiave ominosa già dalla tradizione pagana ${ }^{56}$ per leggervi con ottica escatologica gli indizi luttuosi di un processo irreversibile di estinzione incombente su umani e animali, come si evince dalla chiusa del suo discorso: adeo ut defectura successio et defuturum animantum genus adempto uitalis partus legitimo ordine crederetur.

15 La propensione a concentrarsi su fatti a vario titolo straordinari per trarne prova dell'incessante miseria Romanorum sembra tradire talvolta la percezione da parte dello storico di un legame fra quelli e una conduzione della guerra aliena dal rispetto delle dovute regole. In tal senso si segnala la ripresa di notizie concernenti un episodio accaduto ancora al tempo della guerra contro Taranto, molto probabilmente nel 271 a. C., quando una legione romana, disperando della vittoria, fece trucidare tutti gli abitanti di Reggio meritando la punizione inflitta dal console Genucio che, dopo aver assediato la città, fatti prigionieri tutti coloro che vi si trovavano, inflitti supplizi a disertori e predatori, fece trasferire i soldati colpevoli dell'atto a Roma, dove furono fustigati e decapitati in mezzo al foro per volere del popolo ${ }^{57}$. È indicativo, in particolare, che dopo aver rievocato l'episodio, Orosio riservi un giudizio tagliente alla concezione della giustizia sottesa alla soluzione adottata dai Romani, a suo parere evidentemente colpevoli di considerare una vittoria il risultato ottenuto eliminando una propria legione, laddove qualora fosse andata persa per mano dei nemici non sarebbe stata altro che una sconfitta : Visa sibi est tunc Roma uincere, cum legionem suam integram occidit, quae sine dubio uicta fuisset, si eam hostili proelio perdidisset ${ }^{58}$. È alla luce di quella che appare una considerazione tesa a smascherare l'ambigua etica romana della guerra che deve forse intendersi la registrazione immediatamente successiva di una sequenza di obscena e dira prodigia verificatisi in seguito, nel 269 a. C., nell'urbe e in altre località come Formia e Cales ${ }^{59}$. Nonostante la difficoltà di formulare ipotesi sull'origine delle notizie, la loro attestazione nel passo concorre senz'altro a rimarcare a tinte fosche il periodo considerato, come 
d'altro canto dimostra il riferimento nell'immediato seguito del discorso a fatti del medesimo tenore avvenuti anche l'anno seguente, il $268 \mathrm{~d}$. C., nel corso della campagna condotta dal console Sempronio contro i Picenti. In tale circostanza, stando al racconto di Orosio che in tal caso rielabora materiali di cui si coglie traccia in Frontino ${ }^{60}$ e in Floro ${ }^{61}$, durante una fase di stallo che vedeva contrapposti a breve distanza i due eserciti, ebbe luogo all'improvviso un terremoto che suscitò terrore nelle truppe rendendole inebetite $\mathrm{e}$ tanto sbalordite da non riuscire a passare subito all'azione come convenuto in precedenza ${ }^{62}$. Oltre che per la rielaborazione di indicazioni comunque ricavabili dalla tradizione, già attenta a registrare gli effetti psicologici dell'evento sismico sull'andamento delle operazioni, la "riscrittura" degli eventi proposta da Orosio si segnala ancora una volta per la peculiare valutazione datane e in particolare per la scelta di insistere sulle numerose perdite di vite che lo scontro comportò, malgrado l'esito vittorioso, col fine ultimo di sostenere che la terra avesse tremato emettendo un gran boato nella previsione di dover ricevere una grande quantità di sangue umano: ut merito dicatur tantum humanum sanguinem susceptura etiam cum gemitu horrisono tunc terra tremuisse ${ }^{63}$.

L'inclinazione a concepire una natura vivificata e impegnata a manifestare il proprio dissenso per le azioni umane tramite eventi classificabili in chiave pagana quali presagi, che si ricava da tali osservazioni, può essere colta anche nell'attestazione nell'immediato prosieguo della narrazione ${ }^{64}$ di fatti altrettanto eccezionali come la fuoriuscita di sangue dal suolo e la caduta di latte dal cielo occorsi l'anno successivo, nel 267 a. C., nei quali Orosio sembra identificare di fatto il malaugurante preannuncio dell'esito delle sleali manovre dei Cartaginesi, colpevoli di aver fornito aiuto ai Tarantini contro i Romani, e di aver poi negato l'azione compiuta di fronte all'ambasceria inviata loro dal Senato, dunque responsabili di aver spergiurato oltre che di aver infranto il foedus contratto con Roma. La correlazione fra eventi prodigiosi e accadimenti storici appare in tal caso tanto più rilevante se, tenendo conto dell'assenza di altri riscontri storiografici sull'ambasceria ( LIPPOLD, 1976, I: 434), data l'assenza di altri riscontri storiografici sull'ambasceria ipotizziamo che la notizia vada ascritta direttamente alla mano di Orosio, sicché più in generale se ne dovrà dedurre che con una ponderata "riscrittura", arricchita di nessi e dati introdotti ex nouo, egli mirasse a fornire il quadro di una storia repubblicana dipanatasi nel III sec. a. C. tra azioni inique consumate dai Romani e dai lori rivali e i loro riflessi leggibili nelle calamità scatenate da una natura a tratti concepita come giudice consapevole. Del resto, sotto tale profilo sono emblematiche le osservazioni che accompagnano nel seguito del passo sopra considerato la registrazione di un'altra epidemia pestilenziale esplosa ancora l'anno seguente, nel $266 \mathrm{a}$. C., e protrattasi per più di due anni ${ }^{65}$. A tal proposito, pur mettendo a frutto dati presumibilmente tratti dalla tradizione liviana (LIPPOLD, 1976, I : 434) ${ }^{66}$, rievocata la notizia sull'interpretazione datane dai Libri Sybillini secondo cui essa sarebbe stata provocata dall'ira degli dei, nel tentativo di sottrarsi all'obiezione di aver funzionalmente sottolineato tale aspetto Orosio opta per una eloquente conciliazione tra causalità di ordine scientifico e valorizzazione del ruolo giocato in rapporto ad eventi calamitosi da una volontà superiore che nella sua prospettiva s'incarna nel Dio cristiano ${ }^{67}$.

17 Ancora in relazione al III secolo, segnatamente al periodo precedente l'attacco sferrato da Annibale contro Roma con la II guerra punica, non va trascurata l'attestazione in un passo del IV libro di un serie di dira prodigia manifestatisi prima dello scontro di Gaio Flaminio con i Galli quando nel territorio piceno si vide scorrere sangue in un fiume, in quello toscano prese fuoco il cielo e nell'area di Rimini rifulse una luce improvvisa in piena notte 
e apparvero tre lune distinte in tre punti diversi ${ }^{68}$, in concomitanza con dei sismi che colpirono la Caria e Rodi, ivi provocando perfino la caduta del famoso Colosso. Preceduta dal richiamo ad altri eventi catastrofici anteriori, quali uno straripamento del Tevere e una devastazione dell'urbe ad opera di un incendio propagatosi fino a danneggiare il tempio di Vesta, a proposito dei quali Orosio non esita a sottolineare la portata dirompente e a rimarcare criticamente il mancato intervento degli dei impotenti ad evitare l'estinzione del fuoco sacro ${ }^{69}$, la sua ricostruzione dei fatti straordinari accaduti nel 223 a. C. si segnala per la consueta inclinazione a cumulare eventi in effetti ascrivibili all'arco di un ventennio. Inoltre l'autore non manca di evidenziare puntualmente il fatto che il console Flaminio riportò la vittoria sui Galli dopo che, incurante degli auspici infausti ricavati da quei prodigi, aveva deciso di lanciarsi nella battaglia, notazione nella quale si può leggere una rinnovata critica sull'inadeguatezza delle pratiche augurali.

La tendenza a condensare in modo mirato la successione temporale rilevata per la registrazione di fatti a vario titolo straordinari accaduti nei secoli v-III a.C. appare confermata a grandi linee dai riferimenti a quelli verificatisi nei due secoli successivi, che trovano spazio nel $\mathrm{V}$ libro delle Historiae aduersus Paganos. Se da un canto la possibilità di comparare le attestazioni orosiane relative a tale fase storica con quelle registrate per il periodo in questione da Giulio Ossequente consente di verificare meglio la derivazione e l'adattamento delle notizie, d'altra parte la contrazione dei tempi e le correlazioni stabilite dallo storico tardoantico continuano a documentare una rielaborazione strumentale delle informazioni. In tal senso va considerata innanzitutto la ripresa di un episodio verificatosi sotto il consolato di Lucio Cecilio Metello e Quinto Fabio Massimo Serviliano, ovvero nel 142 a.C. quando - stando alla ricostruzione orosiana-si registrarono vari prodigi fra cui la comparsa di un androgino cui gli aruspici provvidero ordinandone l'annegamento: una misura dall'autore giudicata crudele e di nessun giovamento alla luce della pestilenza scoppiata poco dopo in modo così impetuoso da sterminare presto gli addetti alle sepolture, da riempire di cadaveri e svuotare di vivi le grandi dimore dell'urbe, lasciandole ricchissime ma prive di eredi e da gettare Roma in una condizione di tragico isolamento a causa del fetore dei cadaveri, lasciati a imputridire ovunque, che impediva di avvicinarsi alla città ${ }^{70}$. Tributario di un'indicazione attestata per il medesimo anno anche nel Liber prodigiorum ${ }^{71}$, che tuttavia menziona Luni quale luogo dell'omicidio e dell'epidemia e non pone i due fatti in connessione, Orosio non si limita a rigettare la funzione espiatoria della pratica di soppressione dell'androgino ( abominatio), pur consueta per la prassi pagana ${ }^{72}$, bensì sembra respingerne con ottica cristiana il significato. In particolare, non rinuncia a censurarne l'impietas e infine sostenendo che anziché placare la divinità l'avrebbe piuttosto istigata a colpire successivamente Roma con un contagio capace di provocare la morte di tanti uomini, poi destinato ad esaurirsi non per effetto di alcun sacrificio riparatore bensì grazie ad un arcano giudizio :

Expiatio illa crudelis et uiam mortibus hominum morte hominis struens tandem Romanis inter miserias suas erubescentibus, quam misera et uana esset, innotuit. Ante enim in suffragium praeueniendae cladis est habita, et sic pestilentia consecuta est; quae tamen sine ullis sacrificiorum satisfactionibus tantummodo secundum mensuram arcani iudicii expleta correptione sedata est $^{73}$.

Oltre a chiamare in causa, evidentemente in chiave cristiana, un intervento non lontano da quei giudizi di Dio cui nel corso dell'opera è attribuito un ruolo decisivo in relazione alle vicende romane di età imperiale (MAZZARINo [1959], 2008: $57 \mathrm{ss.)}$ ), la riscrittura orosiana trae in effetti lo spunto da un evento assunto quale presagio dalla mentalità 
romana come la nascita di un androgino per mettere in luce oltre al carattere menzognero delle pratiche espiatorie pagane anche la loro inefficacia, distinguendosi in ciò da fonti pur affini per contenuto come Giulio Ossequente che ne attesta invece l'utilità nel garantire il ripristino della pax deorum ${ }^{74}$. Del resto, che l'interesse dello storico per gli eventi del $142 \mathrm{a}$.C. fosse guidato dalla finalità precipua di criticare il comportamento assunto dai pagani è chiarito dal seguito del suo discorso dove egli si spinge perfino ad accusare gli aruspici di facile propensione a millantare il buon esito dei propri interventi, affermando che qualora avessero svolto cerimonie ufficiali di espiazione durante la fase di regresso del contagio, non avrebbero esitato a rivendicare a sé e alle divinità pagane il ritorno a condizioni di salute: Quam si artifices illi circumuentionum haruspices sub ipsa ut adsolent declinatione morborum forte celebrassent, procul dubio sibi dis et ritibus suis reductae sanitatis gloriam uindicassent ${ }^{75}$. Si tratta di una valutazione che seppur concepita in veste di ipotesi acquista il valore di un atto formale di denuncia della premeditata falsità insita nei rituali pagani, soprattutto se considerata alla luce della desolata rappresentazione di una Roma vittima delle sue credenze superstiziose contenuta nella chiusa del passo : Ita misera et ad sacrilegia male religiosa ciuitas mendaciis, quibus liberari non poterat, ludebatur. In tal senso, non va trascurato il lapidario giudizio da Orosio riservato agli aruspici, aucupatores euentuum et structores fallaciarum in un luogo successivo del medesimo capitolo, a margine di annotazioni riguardanti eventi prodigiosi verificatisi sotto il consolato di Marco Emilio Lepido e Gaio Ostilio Mancino, dunque nel 137 a. C., per fronteggiare i quali non bastarono le consuete misure ${ }^{76}$, considerato l'esito infelice delle operazioni militari più tardi condotte dal secondo dei due, costretto ad un patto infamante con i Numantini ${ }^{77}$. La comparazione con le fonti attestanti il verificarsi nel medesimo torno di anni di eventi straordinari, sia pur di tipo diverso ${ }^{78}$, probabilmente tributarie di notizie di matrice liviana, consente ancora una volta di apprezzare accanto alla cifra personale della riscrittura orosiana dei fatti l'abilità di trarne indizio dell'incongruità delle pratiche religiose pagane.

In quest'ottica merita di essere rilevata anche la rielaborazione in un passaggio successivo del $\mathrm{V} \mathrm{libro}^{79}$ di informazioni riguardanti alcuni eventi eccezionali quali la comparsa a Roma di un bambino deforme, un'eruzione rovinosa dell'Etna in Sicilia e la nascita di messi sugli alberi nell'area di Bologna, assegnati all'anno del consolato di Servio Fulvio Flacco e di Quinto Calpurnio Pisone, ovvero il $135 \mathrm{a}$. C. Al di là della corrispondenza con alcune tipologie di prodigi attribuite da Giulio Ossequente al biennio 136-135 a. C. ${ }^{80}$, la narrazione si segnala per il nesso suggerito fra i fatti prodigiosi menzionati e l'esplodere della guerra servile in Sicilia ${ }^{81}$. Rievocata con toni enfatici che ne evidenziano la natura di fenomeno comunque sciagurato per l'isola in quanto frutto della sua condizione costantemente in bilico tra la tirannide e la libertà sfrenata conquistata dagli schiavi ${ }^{82}$, quest'ultima si profila nell'interpretazione orosiana quale attacco rovinoso portato alla potenza romana, preannunciato da eventi singolari selezionati per restituire ai lettori ancora una volta l'immagine di una Roma pagana di età repubblicana afflitta da mali d'ogni sorta.

Della capacità dello storico di insistere su tale aspetto rivisitando in modo personale dati desunti dalle fonti offre prova anche un passaggio successivo ${ }^{83}$. In tale contesto la rievocazione di una catastrofica invasione di cavallette verificatasi in Africa nell'anno del consolato di Marco Plauzio e Marco Fulvio Flacco, ovvero nel 125 a. C., tributaria di informazioni attestate per il medesimo anno nel Liber prodigiorum ${ }^{84}$ nonché di probabile derivazione liviana ${ }^{85}$, oltre che di possibile ispirazione agostiniana ${ }^{86}$, presuppone d'altro 
canto la conoscenza da parte di Orosio di casi analoghi occorsi ai propri tempi, come lasciano supporre le riflessioni apposte a chiusura del discorso. Cionondimeno, quale che sia la matrice delle indicazioni puntuali dall'autore riportate nel passo per descrivere il flagello recato dalle locustae e infine la loro morte lungo il litorale africano, il suo racconto ci restituisce comunque accanto alla sua compartecipazione per la morte di tante vittime anche la convinzione che si trattò di un evento di proporzioni eccezionali, incomparabile con casi analoghi accaduti in epoche più recenti ${ }^{87}$, una posizione dietro la quale si deve cogliere la volontà di addebitare implicitamente ancora una volta ai limiti di una società aliena dalla fede cristiana la maggior rovinosità delle disgrazie occorsele.

Alla Sicilia, oggetto di interesse non marginale nelle Historiae adversus Paganos ${ }^{88}$, conduce un altro riscontro orosiano relativo ad una calamità naturale che avrebbe interessato ancora la città di Catania all'epoca della conquista delle Baleari, nel 123-122 a. C. ${ }^{89}$. Per quanto coerente anche in tal caso con le informazioni riportate in modo più scarno da Giulio Ossequente ${ }^{90}$, la notizia offre un altro esempio - come è stato suggerito (LIPPOLD, 1976, II : 421) ${ }^{91}$ - della consonanza dello storico con Agostino, per il quale l'eruzione che ricoprì la città di cenere, muovendo a tal punto a compassione i Romani da indurli a condonare per quell'anno ai suoi abitanti il pagamento della decima, non fu che uno dei tanti mali abbatutisi sull'umanità all'epoca del paganesimo.

\section{La correlazione di calamità, prodigi infausti ed accadimenti politici nella ricostruzione dell'ultimo secolo della repubblica}

Nella registrazione di eventi eccezionali accaduti nell'ultimo secolo dell'età repubblicana (133-31 a. C.), la "riscrittura" orosiana sembra accentuarne talora la correlazione con gli accadimenti politici : indicativo in tal senso il riscontro su un prodigium obscenum ac triste occorso nel 114 a. C., dopo la fine della guerra di Mario contro Giugurta, quando la figlia del cavaliere romano Lucio Elvio, durante un viaggio di ritorno da Roma in Apulia con i genitori, essendo stata costretta a lasciare la carrozza e a salire su un cavallo per sfuggire al temporale e rifugiarsi in una casa vicina, fu uccisa improvvisamente da un fulmine e scaraventata per terra senza abiti né monili, in una posa oscena, mentre la bestia giacque morta poco lontano ${ }^{92}$. Il riferimento nell'immediato seguito del passo all'incesto della vestale Emilia e alla violazione del voto di castità di altre due vestali da lei consegnate agli amici del suo seduttore, nonché alla condanna a morte a quelle riservata ${ }^{93}$, non lascia dubbi sul fatto che Orosio cogliesse nel primo episodio il presagio della fine occorsa alle tre vestali, sia pur sulle orme della tradizione recepita anche da Giulio Ossequente ${ }^{94}$. D'altra parte la sua ricostruzione risulta significativamente preceduta dalla notizia sulla triste sorte inflitta negli stessi giorni a Giugurta, strangolato in carcere dove era stato condotto dai Romani dopo esser stato da loro costretto a sfilare con i figli davanti al carro trionfale ${ }^{95}$, sicché si ha il sospetto che lo storico intendesse evocare quasi un nesso di carattere punitivo tra l'atroce misura riservata dai vincitori a quel rivale che aveva saputo vaticinare all'urbe una fine legata al suo crescente degrado morale ${ }^{96} \mathrm{e}$ la terribile vicenda di corruzione e morte riguardante figure considerate simbolo della stabilità $\mathrm{e}$ durata della res publica romana come le vestali.

Ad una fase più travagliata dell'ultima età repubblicana, ovvero al tempo della guerra sociale, in particolare all'anno 91 a. C. conduce invece la sequenza di prodigi registrata in 
un passo successivo del V libro delle Historiae aduersus Paganos dove Orosio annovera eventi straordinari di natura diversificata ${ }^{97}$, quali un globo di fuoco levatosi da settentrione con un boato fragoroso, la fuoriuscita di sangue dal pane verificatasi durante alcuni banchetti ad Arezzo, e ancora la singolare caduta di grandine frammista a pezzi di cocci protrattasi per una settimana, la comparsa di una fiamma dal suolo nel territorio dei Sanniti, nonché di un globo di colore aureo, precipitato dal cielo sulla terra e poi nuovamente direttosi verso Oriente, un fenomeno avvistato da molti Romani in viaggio e probabilmente identificabile con un lampo sismico (sORDI, $1989: 127-129)^{98}$. Al di là della possibilità di individuare scientificamente l'effettiva natura degli eventi attestati nel passo, occorre ancora una volta sottolineare che Orosio riesce ad avvalersi di informazioni su fatti prodigiosi comunque trasmesse dalla tradizione a proposito del bellum Marsicum, come si evince da un accenno di Cicerone ${ }^{99} \mathrm{e}$ dal più puntuale riscontro di Giulio Ossequente ${ }^{100}$, per farne dei mala capaci di terrorizzare Druso, poco dopo ucciso da mano ignota ${ }^{101}$, vale a dire per lasciare immaginare ai lettori l'esistenza di un nesso tra quei prodigia dira e la fine toccata ad un soggetto responsabile ai suoi occhi di aver sobillato alla guerra i latini dopo aver alimentato le loro speranze di ottenere la cittadinanza ${ }^{102}$. Ciò prima di riannodare a tali fatti, praticamente senza soluzione di continuità $^{103}$, l'assassinio del pretore Gneo Servio, consumato ad Ascoli da consociati poi passati ad un'azione più risoluta condannando a morte per decreto tutti i cittadini romani, vale a dire dando inizio agli scontri veri e propri della guerra sociale, secondo il racconto orosiano preceduti ancora una volta da infamissima prodigia che avrebbero preannunciato lo scoppio di una atrocissima pernicies, cui infine fece seguito la sconfitta di Pompeo nella campagna da lui intrapresa contro i Piceni.

\section{Oltre il buio : l'arrivo di Ottaviano a Roma e i segni premonitori della luminosa era cristiana}

25 La stretta correlazione tra eventi prodigiosi e dissidi capaci di minacciare dall'interno la stabilità dell'urbe che caratterizza i casi appena considerati può infine cogliersi anche nella rievocazione dell'ultimo evento calamitoso annotato per l'età repubblicana, vale a dire l'incendio repentino che avrebbe devastato Roma nel 53 a.C. ${ }^{104}$ : un episodio con ogni probabilità attestato dalla tradizione liviana, come induce a credere anche una dichiarazione successiva di Orosio ${ }^{105}$, nonché prodigii loco habitum secondo Giulio Ossequente ${ }^{106}$, tuttavia menzionato nel passo delle Historiae nella semplice ma incisiva veste di accadimento avvenuto in singolare coincidenza con l'esplodere dei contrasti tra Cesare e Pompeo, vale a dire quale fatto storico dall'autore inteso in chiave simbolica più che ominosa, perciò forse meritevole a suo giudizio di essere accostato in un altro luogo dell'opera ad un evento analogo avvenuto contemporaneamente all'attacco dei Goti ${ }^{107}$.

Con l'incendio del 53 a. C. abbattutosi sull'urbe insieme alla guerra che avrebbe cambiato il destino delle istituzioni romane si chiude di fatto la sequela di calamità e prodigi infausti secondo Orosio susseguitisi nella storia di Roma repubblicana : non è casuale che per gli anni seguenti lo storico registri due fenomeni di ben altro significato, ovvero l'improvvisa comparsa in cielo intorno al disco del sole di un cerchio simile ad un arcobaleno il giorno dell'ingresso a Roma di Ottaviano di ritorno da Apollonia e lo sgorgare d'olio da una taberna per un intero giorno in concomitanza con il condono di tutti i debiti contratti dal popolo romano da lui concesso ${ }^{108}$. Interpretati quali segni premonitori dell'inizio di una nuova era assicurata dall'ascesa di Augusto, vale a dire di 
quella in cui avrebbe visto la luce Cristo, il creatore e reggitore di tutto il mondo, l'Unto ${ }^{109}$ , e i Cristiani avrebbero potuto riversarsi copiosamente senza posa fuori dalla Chiesa ${ }^{110}$, i due episodi documentano non soltanto il ruolo di spartiacque da Orosio assegnato ad Ottaviano, ma anche la sua capacità di rileggere e reinterpretare notizie di eventi singolari per trarne in chiave positiva gli indizi di una semeiotica nuova, messa a punto a partire dai modelli recepiti dalla storiografia classica ${ }^{111}$ ma ormai matura per servire ad una rilettura della storia concepita in prospettiva non più pagana.

In tal senso si segnalano anche i riscontri e le osservazioni su calamità e prodigi di segno negativo raccolti per l'età repubblicana nelle Historiae aduersus Paganos: ispirati da posizioni talora affini a quelle espresse da Agostino ${ }^{112}$, essi riflettono comunque l'impegno di Orosio a superare i filtri frapposti dalla storiografia precedente, a suo avviso protesa a dissimulare le numerositates miseriarum. Eloquente al riguardo la dichiarazione formulata in un passo del IV libro, dove la condanna delle fonti anteriori, colpevoli di aver voluto fornire una rappresentazione artificiosamente positiva della storia di Roma precedente all'avvento del Cristianesimo, si unisce peraltro alla consapevolezza di non poter annullare del tutto gli effetti di tali censure :

Ecce continuatim quae et quanta numeramus accidisse annis singulis plurima, inter quos certe raro aut paene nullo nihil triste gestum, et hoc, cum idem scriptores proposito sibi magis laudandi negotio cauerent numerositates miseriarum, ne eosdem quibus haec e de quibus scribebantur offenderent auditoresque suos exemplis praeteritorum terrere potius quam instituere uiderentur. Porro autem nos in ultimo temporum positi mala Romanorum scire non possumus nisi per eos, qui laudauere Romanos. Ex quo intellegi datur, quanta illa fuerint quae studio propter horrorem repressa sunt, cum tanta inueniuntur quae tenuiter inter laudes emanare potuerunt ${ }^{113}$.

È alla luce di tali notazioni che la «definitiva trasformazione in senso cristiano dell'epitome pagana della storia romana » da Orosio attuata con la sua opera ${ }^{114}$ merita di essere considerata un capitolo tutt'altro che secondario della storiografia tardo-antica.

\section{BIBLIOGRAFIA}

ALLELY A., «Les enfants malformés et considérés comme prodigia à Rome et en Italie sous la République ", REA, 105, 2003, p. 127-156.

ALONSO NÚÑEZ J. M., « La metodología histórica de Paulo Orosio », Helmantica, 136-138, 1994, p. 373-379.

AMPIO R., « La concezione orosiana della storia attraverso le metafore del fuoco e del sangue », CCC , 9, 1988, p. 217-236.

ANDRÉ J.-M., " La notion de pestilentia à Rome : du tabou religieux à l'interprétation préscientifique », Latomus, 39, 1980, p. 3-16.

ARNAUD-LINDET M.-P., Orose. Histoires (Contre les Païens), t. I, Livres I-III, Paris, Les Belles Lettres, 1990. 
BARNES C. L. H., Images and insults. Ancient Historiography and the Outbreak of the Tarantine War, Stuttgart, Steiner, 2005.

BERNSTEIN F., Ludi Publici. Untersuchungen zur Entstehung und Entwicklung der öffentlichen Spiele im republikanischen Rom, Stuttgart, Steiner, 1998.

BERTI N., « Catania e le eruzioni dell'Etna nel II secolo a. C. », in M. SORDI (cur.), Fenomeni naturali e avvenimenti storici nell'antichità, Milano, Vita e Pensiero, 1989, p. 87-102.

BESSONE L., " La tradizione epitomatoria latina in età imperiale ", ANRW, 2, 30, 2, 1982,

p. $1230-1263$.

BLOCH R., Les prodiges dans l'Antiquité classique (Grèce, Étrurie et Rome), Paris, Presses Universitaires de France, 1963.

BODSON L., « Le vocabulaire latin des maladies pestilentielles et épizootiques », in G. SABBAH (textes réunis et publiés par), Mémoires $X$ : Le latin médical. La constitution d'un langage scientifique. Réalités et langage de la médecine dans le monde romain, Centre Jean-Palerne, Saint-Étienne, Publications de l'Université de Saint-Étienne 1991, p. 215-241.

BONAMENTE G., « Il metus Punicus e la decadenza di Roma in Sallustio, Agostino e Orosio », GIF, 6, 1975, p. 137-169.

BRACCESI L., Terra di confine. Archeologia e storia tra Marche, Romagna e San Marino, Roma, L'« Erma » di Bretschneider, 2007.

BRANDT H., « Historia magistra vitae ? Orosius und die spätantike Historiographie », in A. GOLTZ, H. LEPPIN, H. SCHLANGE-SCHÖNINGEN (hrg. von), Jenseits der Grenzen. Beiträge zur spätantiken und frümittelalterlichen Geschichtsschreibung, Berlin-New York, De Gruyter, 2009, p. 121-134.

BRISSON L., Le sexe incertain. Androgynie et hermaphroditisme dans l'Antiquité gréco-romaine, Paris, Les Belles Lettres, 2008 (Deuxième édition augmentée d'une postface et d'une bibliographie complémentaire).

CASOLI S., « Su un fenomeno naturale descritto da Plinio (nat. 2, 199) accaduto nel territorio modenese », in L. QUILICI, S. QUILICI GIGLI (a c. di), Urbanizzazione delle campagne nell'Italia antica, Roma, L'« Erma » di Bretschneider, 2001, p. 63-70.

CESA M., " Le historiae adversus paganos di Orosio nel contesto della storiografia tardoantica », in F. E. consolino (a c. di), Forme letterarie nella produzione latina di IV-V secolo. Con uno sguardo a Bisanzio, Roma, Herder, 2003, p. 19-31.

CHAMPEAUX J., « Le Tibre, le pont et les pontifes. Contribution à l'histoire du prodige romain », RÉL , 81, 2003, p. 25-42.

CHASSIGNET M., « Les catastrophes naturelles et leur gestion dans l'ab urbe condita de Tite-Live », Caesarodunum, 39, 2005, p. 337-352.

CORSINI E., Introduzione alle storie di Orosio, Torino, Giappichelli, 1968.

CRACCO RUGGINI L., « L'ordine naturale sconvolto e la morte di un mondo nella storiografia tardoantica », Rivista Storica Italiana, 114, 2002, p. 820-850.

CRIFò G, « "Prodigium” e diritto : il caso dell'ermafrodita », Index, 27, 1999, p. 113-120.

DE FILIPPIS CAPPAI C., «Il culto di Asclepio da Epidauro a Roma : medicina del tempio e medicina scientifica », CCC, 12, 1991, p. 271-284. 
DE SAINT-DENIS E., « Les énumérations de prodiges dans l'oeuvre de Tite-Live », $R P h, 16,1942$, p. 126-142.

DELCOURT M., Stérilités mystérieuses \& naissances maléfiques dans l'Antiquité classique, Paris, 1986.

DIESNER H. J., « Orosius und Augustinus », AAHung, 11, 1963, p. 89-102.

DREWS R., « Pontiffs, Prodigies, and the Disappearance of the Annales Maximi », CPh, 83, 1988, p. 289-299.

DUTOIT E., « Tite-Live s'est-il intéressé à la médecine ? », MH, 5, 1948, p. 116-123.

FABBRINI F., Paolo Orosio, uno storico, Roma, Edizioni di Storia e Letteratura, 1979.

FEVRIER C., « Le bestiaire prodigieux. Merveilles animales dans les littératures historique et scientifique à Rome ", RÉL, 81, 2003, p. 43-64.

FIRPO G., « Osservazioni su temi orosiani (A proposito di alcune recenti pubblicazioni) », Apollinaris , 56, 1983, p. 233-263.

FOWLER W. W., « The Locust-Plague in Africa of 125 B. C. : A Modern Parallel », CR, 18, 1904, p. 394-395.

GARLAND R., The eye of the beholder : deformity and disability in the graeco-roman world, London, Duckworth, $1995\left(2010^{2}\right)$.

GOETZ H. W., Die Geschichtstheologie des Orosius, Darmstadt, Wissenschaftliche Buchgesellschaft, 1980.

GRATTAROLA P., « Il terremoto del 396 e il popolo cristiano di Costantinopoli », in M. SORDI (cur.), Fenomeni naturali e avvenimenti storici nell'antichità, Milano, Vita e Pensiero, 1989, p. 237-249.

GRMEK M. D., «Les vicissitudes des notions d'infection, de contagion et de germe dans la médecine antique ", in G. SABBAH (éd. par), Mémoires V : Textes Médicaux Latins Antiques, Saint-Étienne, Publications de l'Université de Saint-Étienne, 1984, p. 53-70.

HAUG I., « Der römischen Bundesgenossenkrieg 91-88 v. Chr. bei Titus Livius », WJA, 2, 1947, p. $100-139 ; 201-258$.

JIMÉNEZ DELGADO J. (a), « Importancia de los prodigios en Tito Livio », Helmantica, 12, 1961, p. 27-46. JIMÉNEZ DELGADO J.(b), « Classifición de los prodigios titolivianos », Helmantica, 12, 1961, p. 441-461. JIMÉNEZ DELGADO J., « Postura de Livio frente al prodigio », Helmantica, 14, 1963, p. 381-419.

KRAUS F. B., An interpretation of the omens, portents and prodigies recorded by Livy, Tacitus and Suetonius, Philadelphia, University of Pennsylvania, 1930.

LACROIX B., Orose et ses idées, Montréal-Paris, Vrin, 1965.

LEBRECHT SCHMIDT P. L., « Iulius Obsequens und das Problem des Livius-Epitome. Ein Beitrag zur Geschichte der lateinischen Prodigienlitteratur ", AAWM, 5, 1968, p. 155-242.

LEVENE D. S., Religion in Livy, Leiden, Brill, 1993.

LIPPOLD A., « Der erste Punische Krieg bei Orosius », RhM, 97, 1954, p. 254-286.

LIPPOLD A., Orosio. Le storie contro i Pagani, Milano, Lorenzo Valla, 1976.

LIZZI TESTA R., "Quando nella curia furono viste fiorire le scope : il Senato di Valentiniano », in EAD. (cur.), Le trasformazioni delle élites in età tardo-antica, Roma, L'« Erma » di Bretschneider, 2006, p. 239-276. 
LORSCH WILDFANG R., Rome's Vestal Virgins. A Study of Rome's Vestal priestesses in the late Republic and early Empire, New York, Routledge, 2006.

MACBAIN B., Prodigy and Expiation : A Study in Religion and Politics in Republican Rome, Brussels, Latomus, 1982.

MARCHETTA A., « Aspetti della concezione orosiana della storia », in G. URSO (cur.), Hispania terris omnibus felicior. Premesse ed esiti di un processo di integrazione. Atti del Convegno Internazionale, Cividale del Friuli, 27-29 settembre 2001, Pisa, ETS, 2002, p. 323-343.

MARCONE A., « Il sacco di Roma del 410 nella riflessione di Agostino e di Orosio », RSI, 114, 2002, p. 851-867.

MARTÍNEZ CAVERO P., « Signos y prodigios. Continuidad e inflexión en el pensamiento de Orosio ", Antig. crist. (Murcia), 14, 1997, p. 83-95.

MAZZARINO S., La fine del mondo antico. Le cause della caduta dell'impero romano, Milano, Garzanti, 1959, rist. Torino, Bollati Boringhieri, 2008.

MILES G. B., Livy. Reconstructing Early Rome, Ithaca, Cornell University Press, 1995.

MOMIGLIANO A., «Storiografia pagana e cristiana nel secolo IV d. C. », in ID. (a c. di), Il conflitto fra paganesimo e cristianesimo nel secolo IV, Torino, Einaudi, 1975, p. 89-110.

MUSIAL D., « Sur le culte d'Esculape à Rome et en Italie », DHA, 16, 1990, p. 231-238.

NUFFELEN VAN P., Orosius and the Rhetoric of History, Oxford, University Press, 2012.

OAKLEY S. P. (ed.), A Commentary on Livy. Books VI-X, vol. II Books VII-VIII, Oxford, Clarendon Press, 1998.

OGILVIE R. M., A Commentary on Livy. Books 1-5, Oxford, Clarendon Press, 1965.

PASCHOUD F., « La polemica provvidenzialistica di Orosio », La Storiografia ecclesiastica nella Tarda antichità. Atti del Convegno tenuto in Erice (3-8, XII 1978), Messina, Centro di Studi Umanistici, 1980, p. 113-133.

PISI G., La peste in Seneca tra scienza e letteratura, Roma, Bulzoni, 1989.

POLICHETTI A., Le « Historiae » di Orosio e la tradizione imperiale nella « storiografia ecclesiastica » occidentale (311-417), Napoli, Edizioni Scientifiche Italiane, 2000.

PRANDI L., Callistene uno storico tra Aristotele e i re Macedoni, Milano, Jaka Book, 1984.

PRANDI L., « La rifondazione del "Panionion” e la catastrofe di Elice (373 a. C.) », in M. SORDI (cur.), Fenomeni naturali e avvenimenti storici nell'antichità, Milano, Vita e Pensiero, 1989, p. 43-58.

RÁBADE NAVARRO M.-A., « Los dioses y la religión romana en las historias de Paulo Orosio », Habis, 27, 1996, p. 225-234.

RAWSON E., « Prodigy lists and the use of the Annales Maximi », CQ, 21, 1971, p. 158-169 (rist. in Ead., Roman Culture and Society, Oxford, Clarendon Press, 1991, p. 1-15).

ROSENBERGER V., Gezähmte Götter. Das Prodigienwesen der römischen Republik, Stuttgart, Steiner, 1998.

ROSENBERGER V., « Prodigien aus Italien : geographische Verteilung und religiöse Kommunikation », CCG, 16, 2005, p. 235-257.

RosSBACH 0., « Der Prodigiorum liber des Iulius Obsequens », RhM, 52, 1897, p. 1-12. 
SALVO D., « La Sicilia e la Spagna nelle Storie di Paolo Orosio », in P. ANELLo, J. MARTínEZ-PINNA (eds.), Relaciones interculturales en el mediterráneo antiguo : Sicilia e Iberia, Málaga-Palermo, Centro de Ediciones de la Diputación de Málaga, 2008, p. 233-243.

SANDERS H. A., Die Quellencontamination im 21. und 22. Buche des Livius, Berlin, Mayer \& Müller, 1898. SANTINI C., "L'Adversus Paganos di Orosio e la tradizione manoscritta del Breviarium di Eutropio ", GIF, 9, 1978, p. 79-91.

SANTINI C., « Letteratura prodigiale e sermo prodigialis in Giulio Ossequente », Philologus, 132, 1988, p. $210-226$.

SCARDIGLI B., I trattati romano-cartaginesi, Pisa, Scuola Normale Superiore, 1991.

SCARDIGLI B., « Die Frau im Prodigienwesen in der römischen Republik », in F. BERTHOLET, A. BIELMAN SÀNCHEZ, R. FREI-STOLBA (éds.), Egypte - Grèce - Rome. Les différents visages des femmes antiques. Travaux et colloque du séminaire d'épigraphie grecque et latine de l'IASA 2002, Bern, Peter Lang, 2008, p. 197-222.

SINISCALCO P., «Le sacré et l'expérience de l'histoire : Ammien Marcellin et Paul Orose », BAGB, 4, 1989, p. 355-366.

SORDI M., « Alessandro e Roma nella concezione storiografica di Orosio », Studi Tardoantichi 1, 1986, p. 183-193 (rist. in Ead., Scritti di Storia romana, a c. di C. BEARZoT e G. ZECCHINI, Milano, Vita e Pensiero, 2002, II, p. 423-431).

SORDI M., « Il lampo sismico del 91 a. C. e la denunzia dei cavalieri contro Druso », in M. Sordi (cur.), Fenomeni naturali e avvenimenti storici nell'antichità, Milano, Vita e Pensiero, 1989, p. 127-132. STEELE R. B., « The Pestilences mentioned by Livy », TAPhA, 33, 1902, p. LXIV-LXV.

STOK F., « Livio e la peste di Siracusa », Schol(i)a, 2, 1999, p. 23-43.

STOK F., « Il lessico del contagio », in P. RADici colace, A. zUMBo (cur.), Atti del Seminario Internazionale di Studi Letteratura scientifica e tecnica greca e latina, Messina, Edas, 2000, p. 55-89. TRAINA G., « Terremoti e società romana : problemi di mentalità e uso delle informazioni ", ASNP, 15,1985 , p. $867-887$.

TRAINA G., « Tracce di un'immagine : il terremoto fra prodigio e fenomeno », in E. GUIDOBONI (dir.), I terremoti prima del Mille in Italia e nell'area mediterranea. Storia, archeologia, sismologia, Bologna, S.G.A., 1989, p. 104-114.

TRAINA G., « Celio Antipatro e il terremoto del 217 a.C. (fr. 20 Peter) », Eutopia, 5, 1996, p. 59-65. WILliam RaSmussen S., Public portents in Republican Rome, Roma, L'« Erma » di Bretschneider, 2003. WOODS D., « An earthquake at Constantinople: Augustine's De excidio urbis VI, 7 ", Augustiniana, 42, 1992, p. 331-337.

ZECCHINI G., Ricerche di storiografia latina tardoantica, Roma, L'« Erma » di Bretschneider, 1993. ZECCHINI G., Il pensiero politico romano. Dall'età arcaica alla tarda antichità, Roma, La Nuova Italia scientifica, 1997. 


\section{NOTE}

1. Sull'opera di Orosio e la sua concezione storiografica, oggetto di un'ampia bibliografia, per un inquadramento generale vd. CORSINI, 1968; FABBRINI, 1979; GOETZ, 1980; FIRPO, 1983 ; ALONSO NÚÑEZ, 1994 ; POLICHETTI, 2000, NUFFELEN VAN, 2012.

2. Oros. I Prol. 9 : Praeceperas mihi uti aduersus uaniloquam prauitatem eorum, qui alieni a ciuitate Dei ex locorum agrestium conpitis et pagis pagani uocantur siue gentiles quia terrena sapiunt, qui cum futura non quaerant, praeterita autem aut obliuiscantur aut nesciant, praesentia tamen tempora ueluti malis extra solitum infestatissima ob hoc solum quod creditur Christus et colitur Deus, idola autem minus coluntur, infamant (Per i passi dell'opera citati nella presente nota nonché infra in quelle seguenti e supra nel testo del contributo si è fatto ricorso all'edizione LIPPOLD, 1976). Di un analogo tentativo di scagionare i cristiani sottolineando il continuo susseguirsi di mali e sventure già in età pagana offre riscontro anche la tradizione anteriore : Cf. Tert. apol. 40-41; Arn. adu. nat. I, 13 ; Cypr. ad Demetr. 2-3.

3. Oros. I Prol. 10.

4. Sulla attendibilità del riferimento di Orosio all'invito a comporre l'opera ricevuto da Agostino si registrano pareri discordi: a fronte di quanti reputano sostanzialmente fededegna la dichiarazione dello storico (e.g. DIESNER, 1963 ; LACROIX, 1965 : 42 ; LIPPOLD, 1976, I : XXI ; FABBRINI, 1979: 70-72; MARTÍNEZ CAVERO, 1997: 85, nonché MARCHETTA, 2002: 335-339), si segnala la posizione di quanti considerano con scetticismo le affermazioni orosiane, o le ammettono parzialmente leggendovi un tentativo dello storico di avvalersi del nome di Agostino per accreditare il proprio progetto o ancora notano comunque la mancata corrispondenza fra il precetto del maestro e la risposta data dal discepolo con le sue Historiae (CORSINI, 1968 : 15 ss., 35-51, 202 ss. ; PASCHOUD, 1980 : 114 ; CESA, 2003 : 29 ; ARNAUD-LINDET, 1990 : XX ss. ; POLICHETTI, $2000: 4-12$; MARCONE, $2002: 859-860$ ).

5. Su tale carattere peculiare dell'opera hanno richiamato l'attenzione ( SINISCALCO, 1989: 361-362; Cracco RUGGINI, 2002 : 822 ; Lizzi TESTA, 2006 : 252-253; per una disamina più specifica cf. MARTÍNEZ CAVERO, 1997).

6. L'influenza dell'opera liviana e in particolare l'inclinazione di Orosio a riprenderne le informazioni su calamità e sventure in modo tendenzioso è lucidamente evidenziata da ZECCHINI ( 1997 : 154)

7. Sui contenuti della trattazione, la tecnica di registrazione di eventi straordinari seguita dall'autore e il suo rapporto con l'opera di Livio vd. soprattutto ROSSBACH, 1987 ; SANTINI, 1988 ; nonché LEBRECHT SCHMIDT, 1968 ; BESSONE, 1982 : 1242-1244.

8. Per una disamina sul punto vd. FABBRINI, 1979: 100-109; ARNAUD-LINDET, 1990 : XXV-XXIX ; POLICHETTI, 2000 : 4-18; con particolare riguardo ad Eutropio vd. SANTINI (1978).

9. Oros. II, 12,1 .

10. Oros. II, 12, 2-4 : Romae ergo post Urbem conditam anno CCLXL suspenso ad modicum bello grauis pestilentia, quae semper ibi raras indutias aut factas intercepit aut ut fierent coegit, per universam ciuitatem uiolenter incanduit, ut merito praecedente prodigio caelum ardere uisum sit, quando caput gentium tanto morborum igne flagravit. Nam eo anno Aebutium et Seruilium ambo consules pestilentia consumpsit, militares copias plurima ex parte confecit, multos nobiles praecipueque plebem foeda tabe deleuit; quamuis iam etiam superiore quarto anno oborta lues eundem populum depopulata sit.

11. Liv. III, 5, 14 : Vt Romam reditum est et iustitium remissum, caelum uisum est ardere plurimo igni, portentaque alia aut obuersata oculis aut uanas exterritis ostentauere species... 
12. Sullo spazio riservato nell'opera liviana alla registrazione di eventi calamitosi e a vario titolo prodigiosi, fra diversi contributi oltre KRAUS, 1930 Cf. soprattutto de SAINT-DENIS, 1942 ; JIMÉNEZ DELGADO, 1961a, 1961b, 1963 ; LEVENE, 1993 : 16-30 ; CHASSIGNET, 2005.

13. Di tale prassi offre testimonianza Liv. XLIII, 13; sull'argomento cf. RAWSON, 1971 (1991); DREWS, 1988; nonché ROSENBERGER, 1998: 161-170; per una lista dei prodigi occorsi in età repubblicana vd. MACBAIN, 1982: 82-106; con particolare riguardo alla loro localizzazione geografica Rosemberger 2005.

14. Liv. III, 6, 2-3: Graue tempus et forte annus pestilens erat urbi agrisque, nec hominibus magis quam pecori, et auxere uim morbi, terrore populationis pecoribus agrestibusque in urbem acceptis. Ea conluuio mixtorum omnis generis animantium et odore insolito urbanos et agrestem confertum in arta tecta aestu ac uigiliis angebat, ministeriaque in uicem ac contagio ipsa uolgabant morbos...

15. Sull'argomento, oltre i rilievi sintetici di STEELE, 1902, cf. DUTOIT, 1948 ; ANDRÉ, 1980 ; STOK, 1999.

16. Interpretato da Orosio come premonizione della pestilentia successiva, l'ardere caelum risulta opportunamente definito in senso tecnico prodigium se consideriamo l'accezione di quest'ultimo termine fornita da Cic. Diu. I, 93.

17. Nel passo orosiano va notata l'attestazione accanto al termine pestilentia di voci quali tabes e lues sulla cui valenza tecnica cf. BODSON, 1991 : 220-221 ; sTOK, 2000 : 62-63.

18. Sen. Nat. Quaest. I, 15, 5 : Inter haec licet ponas et quod frequenter in historiis legimus, caelum ardere uisum, cuius nonnumquam tam sublimis ardor est ut inter sidera ipsa uideatur, nonnumquam tam humilis ut speciem longinqui incendii praebeat; per ulteriori attestazioni tecniche della locuzione ardere caelum vd. Jul. Obs. $14 ; 15 ; 20 ; 38 ; 51$.

19. Per una disamina al riguardo vd. AMPIO, 1988.

20. Dionys. Hal. Ant. Rom. IX, 67, 3 dove figura un'accurata descrizione sull'iniziale dilagare del contagio fra diverse specie animali in ambito rurale e il successivo spostamento dell'epidemia nell'urbe, corredata da indicazioni sulle diverse perdite registratesi, sulle modalità di sepoltura, sul periodo dell'anno in cui sarebbe insorta nonché sulla sua durata, probabilmente ispirata da interessi specifici dello storico, come porta a ritenere anche l'attenzione riservata ad un episodio analogo in Ant. Rom. X, 53, 3. Sulla maggiore precisione dionisiana, imputabile alla puntualità delle fonti annalistiche compulsate dall'autore, ha posto l'accento ANDRÉ, 1980 : 12-13.

21. Significativo ciò che si legge a proposito della facile propensione a lasciarsi spaventare da eventi straordinari soprattutto in tempi di guerra : in Cic. Diu. II, 58 : Atque haec in bello plura et maiora uidentur timentibus, eadem non tam animaduertuntur in pace; accedit illud etiam, quod in metu et periculo cum creduntur facilius, tum finguntur impunius ; vd. inoltre Sen. Nat. Quaest. VI, 29, 3.

22. Liv. III, 5, 14 : His auertendis terroribus in triduum feriae indictae, per quas omnia delubra pacem deum exposcentium uirorum mulierumque turba implebantur.

23. Oros. III, 4, 1-2: Anno ab urbe condita CCCLXXXIIII L. Genucio et Q. Seruilio consulibus ingens uniuersam Romam pestilentia corripuit : non ut adsolet plus minusue solito temporum turbata temperies, hoc est aut intempestiua siccitas hiemis aut repentinus calor ueris aut incongruus umor aestatis uel autumni diuitis indigesta inlecebra, insuper etiam exspirata de Calabris saltibus aura corrumpens, repentinos acutarum infirmitatum adferre transcursus...

24. Oros. III, 3, 1-2 ; per la disamina dei fatti occorsi in tale area e l'analisi delle fonti si veda PRANDI, 1984 : 42 ss. ; EAD., 1989.

25. Aug. De excidio urbis Romae sermo, 7 (PL, XL, cc. 722-723 ss.) dove, pochi anni dopo l'attacco di Alarico a Roma del 410, l'autore, impegnato a sostenere che Dio intendesse con esso spingere Roma ad emendarsi ma non volesse distruggerla, ricorda che anche Costantinopoli aveva subito un analogo avviso tramite il terremoto ; in proposito cf. anche wOoDs, 1992, nonché GRATTAROLA, 1989.

26. Liv. VII, 1, 7 ; 2-3 e in proposito vd. OAKLEY, 1998 : 34-40. 
27. Sulle modalità di registrazione di sismi in età repubblicana e la loro valutazione in termini di prodigio vd. Inoltre TRAINA, $1985: 871-872 ; 1989: 109$.

28. Si consideri in Oros. III, 4, 3 il riferimento all'attacco portato dalla tabes in modo indifferenziato a individui di ogni genere, età e condizione sociale.

29. Sulle teorie classiche in tema di contagio cf. Lucr. IV, 816-817 ; VI, 662-664; 769-772 ; Varro, Rust. I, 12 ; I, 4, 4 ; Vitr. I, 4, 1 ; Sen. Nat. Quaest. III, 202 ; VI, 28 ; Plin. II, 107, nonché ANDRÉ, 1980 ; GRMEK, 1984 ; PISI, 1989 : 31-43 ; STOK, 2000 : 76-89.

30. Oros. III, 4, 5 : Cum pestilentia in dies crudesceret, auctores suasere pontifices, ut ludi scaenici diis expetentibus ederentur ; in proposito vd. anche BERNSTEIN, 1998 : 119-120.

31. Oros. III, $4,4$.

32. Oros. III, $5,1$.

33. Utile rileggere in tal senso il pesante giudizio espresso sui ludi da Aug. De civ. I, 32 : ludi scaenici, spectacula turpitudinum et licentia uanitatum, non hominum uitiis, sed deorum uestrorum iussis Romae instituti sunt... Dii propter sedandam corporum pestilentiam ludos sibi scaenicos exhiberi iubebant ; pontifex autem propter animorum cauendam pestilentiam ipsam scaenam constitui prohibebat... Neque enim et illa corporum pestilentia ideo conquieuit, quia populo bellicoso et solis antea ludis circensibus adsueto ludorum scaenicorum delicata subintrauit insania ; sed astutia spirituum nefandorum praeuidens illam pestilentiam iam fine debito cessaturam aliam longe grauiorem, qua plurimum gaudet, ex hac occasione non corporibus, sed moribus curauit inmittere...

34. Oros. III, $4,4$.

35. Oros. III, 5, 1-2: Sequitur hanc miseram luem miserioremque eius expiationem proximo anno satis triste prodigium. Repente siquidem medio urbis terra dissiluit, uastoque praeruptu hiantia subito inferna patuerunt. Manebat diu ad spectaculum terroremque cunctorum patenti uoragine inpudens specus nefariamque uiui hominis sepulturam diis interpretibus expetebat.

36. Oros. III, $5,3$.

37. Liv. I, 12, 10 e in proposito Ogilvie 1965, pp. 75-78. Per ulteriori attestazioni della medesima versione dei fatti cf. anche Dionys. Hal. II, 42 ; Plut. Rom. 18.

38. Liv. VII, 6, 1-6 ; sul passo si veda il commento di MILES, 1995 : 35-38 che sottolinea la difficoltà dello storico di orientarsi fra tradizioni diverse riguardanti la fase più antica della storia romana ; ulteriori precisazioni sull'episodio anche in WILLIAM RASMUSSEN, 2003 : 38-40.

39. Oros. III, 7, 4: Tunc etiam nox usque ad plurimam diei partem tendi uisa est et saxea de nubibus grando descendens ueris terram lapidibus uerberauit.

40. Che pur ricordando l'analogia con Liv. VII, 28, 7 esprime tuttavia dubbi sulla derivazione da quest'ultimo della notizia orosiana.

41. Liv. VII, 28, 7 : Prodigium extemplo dedicationem secutum, simile uetusto montis Albani prodigio; namque et lapidibus pluit et nox interdiu uisa intendi... e in proposito la disamina delle diverse possibilità di identificazione dell'episodio in OAKLEY, $1998: 269-270$.

42. Eloquente, del resto, l'affermazione che si legge in Oros. III, 7, 1 : Numerandum etiam inter mala censeo primum illud ictum cum Carthaginiensibus foedus, quod isdem temporibus fuit; praesertim ex quo tam gravia orta sunt mala, ut exim coepisse uideantur... Quanto al significato storiografico della notizia orosiana da porsi in relazione con Diod. Sic. XVI, 69, 1, vd. SCARDIGLI, 1991 : 95-99.

43. Oros. III, 7, 3: Quem ingressum Carthaginiensium in Italiam malorum grandinem secuturam continuarumque miseriarum tenebras iuges historiarum fides locorumque infamia et abominatio dierum, quibus ea gesta sunt, protestantur.

44. Oros. III, 7, 5 : Quibus diebus etiam Alexander Magnus, uere ille gurges miseriarum atque atrocissimus turbo totius Orientis, est natus; sull'equiparazione della nascita di Alessandro a eventi catastrofici e più in generale sulla posizione ostile dello storico nei riguardi del Macedone vd. soRDI, 1986. 
45. Si ricordi il giudizio negativo sulla fine di Cartagine, prodromica dell'avvio della decadenza per Roma, in Oros. IV, 23, 9-10; sulla peculiare lettura orosiana delle guerre puniche utili precisazioni in LIPPOLD, 1954 ; BONAMENTE, 1975 : 161-169; FABBRINI, $1979: 226-233$ et 247-250.

46. Oros. III, 22, 1 ; 3-4: Sequitur annus, quo Romani instaurato a Samnitibus bello uicti sunt atque in castra fugerunt... Aduersum quos Papirius consul cum exercitu missus cum a pullariis auguribus uana coniectantibus congredi prohiberetur, inridens eos tam feliciter confecit bellum quam constanter arripuit. Nam in hoc proelio XII milia hostium caesa, III milia capta referuntur. Sed hanc quoque istius uere laudandam uictoriam, quam uani auspices impedire non potuerunt, oborti subito corrupere morbi.

47. Come evidenziato da LIPPOLD (1976, I : 426) si può rilevare il diverso riscontro dei fatti dato da Liv. X, 40, 2-5 ; 9-14 da cui emerge che nel 293 a. C. Papirio si sarebbe accinto allo scontro dopo che gli auspices, condividendo l'entusiasmo delle truppe gli avevano falsamente annunciato un presagio favorevole; successivamente, pur essendo venuto a conoscenza della verità, egli avrebbe deciso di proseguire nella sua impresa, attenendosi a quanto gli era stato comunque vaticinato. Della notizia si trova indicazione anche in Val. Max. VII, 2, 5.

48. Oros. III, 22, 5 : Nam tanta ac tam intolerabilis pestilentia tunc corripuit ciuitatem, ut propter eam quacumque ratione sedandam libros Sibyllinos consulendos putarint horrendumque illum Epidaurium colubrum cum ipso Aesculapi lapide aduexerint : quasi uero pestilentia aut ante sedata non sit aut post orta non fuerit.

49. Si veda Liv. X, 47, 7 secondo cui il fra il 292 e il 291 a. C., essendosi abbattuta un'epidemia sull'urbe, per fronteggiare il pericolo si decise di consultare i Libri Sibillini da cui si apprese che occorreva far venire da Epidauro a Roma il dio Esculapio; data l'impossibilità di procedere a ciò a causa della guerra, si optò per la celebrazione di una supplicazione di un giorno al dio. Il preciso riferimento al serpente contenuto nel passo orosiano indurrebbe tuttavia a ipotizzare che esso sia derivato dalla lettura di dati conservati dalla tradizione epitomata: cf. infatti Liv. Per. XI, 3 secondo cui, in seguito all'epidemia, alcuni ambasciatori incaricati di trasferire da Epidauro a Roma la statua di Esculapio avrebbero riportato in patria il serpente che si riteneva fosse incarnazione del dio, che sarebbe poi sceso sull'isola Tiberina nel luogo in cui fu edificato il tempio di Esculapio ; per ulteriori attestazioni vd. anche la disamina delle fonti in MUSIAL, 1990 ; DE FILIPPIS CAPPAI, 1991 : 275-277.

50. Come sinteticamente proposto da LIPPOLD, 1976, I : 426 il giudizio dello storico merita di essere confrontato con quello espresso da Aug. De civ. III, 17, 2 ; per una notazione analoga cf. anche RÁBADE NAVARRO, 1996 : 228-229 per cui tuttavia in Orosio prevale soprattutto la convinzione che solo la provvidenza possa assicurare la risoluzione dei malanni. Va tuttavia notato che a fronte dell'argomentazione di Agostino, secondo cui l'introduzione del culto avrebbe dovuto essere imputata all'incapacità di Giove di dedicarsi all'apprendimento della medicina a causa delle sue distrazioni in campo amoroso, la riflessione orosiana abbandona il piano della ridicolizzazione della mitologia pagana preferendo insistere sulla inefficacia considerata sulla lunga durata.

51. Per qualche osservazione di carattere più generale sull'uso orosiano delle fonti riguardanti la guerra tarantina vd. BARNES, 2005 : 124-128.

52. Oros. IV, 1, 16-18: Interea Romanus exercitus, postquam uictus clam fugit e castris, miserabilem belli cladem grauioribus monstris auctam accumulatamque persensit. Nam pabulatores forte progressos uelut hostilis quaedam oborta tempestas cum horribili fragore caeli correptos diris fulminibus exussit. Quippe XXX et IIII eorum idem turbo prostrauit; duo et uiginti semineces relicti, iumenta exanimata et capta conplurima : ut merito contigisse non in signum uastationis futurae sed uastatio ipsa referatur.

53. Oros. IV, 2,1 .

54. Oros. IV, 2, 2 : Nam Fabio Gurgite iterum C. Genucio Clepsina consulibus pestilentia grauis urbem ac fines eius inuasit; quae cum omnes tum praecipue mulieres pecudesque corripiens necatis in utero fetibus futura prole uacuabat, et immaturis partubus cum periculo matrum extorti abortus proiciebantur... 
55. Per tale ipotesi vd. LIPPold (1976, I : 432), che rimanda ad Aug. De ciu. III, 17, accogliendo l'ipotesi di una derivazione di entrambi da un'epitome dell'opera liviana avanzata da SANDERS, $1898: 19$ ss.

56. Per qualche approfondimento al riguardo (DELCOURT, 1986 ; ALLELY, 2003 : 139-143).

57. Oros. IV, 3, 3-5. Per ipotesi diverse di datazione dell'episodio vd. LiPPoLD, 1976, I : 433.

58. Oros. IV, $3,6$.

59. Si veda Oros. IV , 4, 1-4 che con dovizia di particolari ricorda la distruzione a causa di un fulmine del Tempio della Salute, il danneggiamento di una parte delle mura situate nella medesima area, l'ingresso a Roma sul far dell'alba di tre lupi che trascinavano un cadavere e spaventati dalle grida dei presenti nel foro vi lasciarono i resti, e ancora il rogo e l'abbattimento delle mura di Formia a seguito della caduta di fulmini, l'apertura a Cales di una voragine da cui sarebbe fuoriuscita una fiamma che, divampando per tre giorni e tre notti, fece ardere un terreno di cinque iugeri rendendolo improduttivo, provocando la distruzione non solo delle messi ma anche di tutte le piante fino alle radici.

60. Front. Strat. I, 12, 3 secondo cui il console T. Sempronio Gracco riuscì ad attaccare e vincere i Picenti solo dopo aver incoraggiato i suoi, spaventati dal terremoto verificatosi prima che avesse inizio lo scontro e averli spronati ad attaccare i nemici, terrorizzati per la superstizione a causa della scossa tellurica : ...cum subitus terrae motus utrasque partes confudisset, exhortatione confirmauit suos et impulit consternatum superstitione inuaderent hostem, adortusque deuicit.

61. Flor. I, 14.

62. Oros. IV, 4, 5-6.

63. Oros. IV , $4,7$.

64. Oros. IV, 5, 1-2: Anno ab urbe condita CCCCLXXX inter multa prodigia sanguis e terra, lac uisum est manare de caelo. Nam et plurimis locis scaturiens e fontibus cruor fluxit et de nubibus guttatim in speciem pluuiae lacte demisso, diri, ut ipsis uisum est, terram imbres inrigauerunt. Eo tempore Carthaginienses dato aduersum Romanos auxilio Tarentinis, cum a senatu per legatos arguerentur, turpissimam rupti foederis labem praesumpto accumulauere peiurio.

65. Oros. IV, 5, 6-7.

66. Che nel riferimento orosiano al censimento compiuto dopo la pestilentia per accertare il numero dei sopravvissuti vede un indizio della ripresa di Liv. Per. XVI (anche se non vi si accenna all'epidemia) ed Eutrop. II, 18.

67. Oros. IV, 5, 8 : Sed, ne quemquam quasi temptatae cauillationis offendat, quod, cum Sybilla iratos deos dixerit, nos iram caelestem dixisse uideamur, audiat et intellegat quia haec, etsi plerumque per aerias potestates fiunt, tamen sine arbitrio omnipotentis Dei omnino non fiunt.

68. Oros. IV, 13, 12-13 e in parallelo Plut. Marc. 4 ; per l'analisi in chiave storiografico-politica delle diverse versioni riportate dalle fonti si veda soprattutto BRACCESI, $2007: 149-152$.

69. Per gli eventi accaduti nel 241-240 a. C., sotto il consolato di T. Lutazio Catulo e A. Manlio, cf. Oros. IV, 11, 6-9, ove risuona in fine l'attacco agli dei pagani: ...et ne sibi quidem dis subuenientibus ignem illum, qui aeternus putabatur, temporarius ignis oppressit. Sull'interpretazione delle inondazioni del Tevere quale avvertimento divino di natura infausta vd. CHAMPEAUX, 2003.

70. Oros. V, 4, 8-9: L. Caecilio Metello Q. Fabio Maximo Seruiliano consulibus inter cetera prodigia androgynus Romae uisus iussu haruspicum in mare mersus est. Sed nihil impiae expiationis procuratio profecit; nam tanta subito pestilentia exorta est, ut ministri quoque faciendorum funerum primum non sufficerent deinde non essent; iamque etiam magnae domus uacuae uiuis plenae mortuis remanserunt: largissimae introrsum hereditates et nulli penitus heredes. Denique iam non solum in urbe uivendi sed etiam adpropinquandi ad urbem negabatur facultas, tam saeui per totam urbem tabescentium sub tectis atque in stratis suis cadauerum putores exhalabantur.

71. Jul. Obs. 22 : Lunae androgynus natus praecepto aruspicum in mare deportatus : tanta fuit Lunensibus pestilentia, ut iacentibus in publicum passim cadaueribus qui funerarent defuerint. 
72. Per qualche altro esempio relativo all'età repubblicana oltre alle testimonianze di Liv. XXVII, 11, 4 ; XXVII, 37, 5 ; XXXI ; 12, 6 ; XXXIX, 22, 5, cf. Jul. Obs. 27a (133 a. C.) ; 32 (122 a. C.) ; 34 (119 a. C.) ; 36 ( 117 a. C.) ; 47 (98 a. C.) ; 48 (97 a. C.) ; 50 (95 a. C.), in proposito vd. inoltre MACBAIN, 1982 :127-135 ; GARLAND, 1995 : 67-72 ; ALLELY, 2003 : 136-139; CRIFÒ, 1999 : 113-115 ; BRISSON, 2008² : 29-32. Più in generale per la valutazione di casi di nascite di neonati malformati, ermafroditi o creature di aspetto misto in età mediorepubblicana quali manifestazioni funeste da superare tramite atti espiatori vd. anche BLOCH, $1963: 73$ ss.

73. Oros. V, $4,10$.

74. Si veda ad es. il riferimento al ritorno della pax dopo le misure prese in seguito alla comparsa di ermafroditi in Jul. Obs. $36 ; 50$.

75. Oros. V, $4,11$.

76. Oros. V, 4, 19: M. Aemilio Lepido C. Hostilio Mancino consulibus prodigia apparuere diuersa et, quantum in ipsis fuit, ex more curata sunt. Sed non semper aucupatoribus euentuum et structoribus fallaciarum haruspicibus opportuni casus suffragantur.

77. Oros. V, 4, 20.

78. Liv. Per. LV, 6-7 ; Jul. Obs. 24 ; Val. Max. I, 6, 7 e inoltre Min. Fel. Octav. 26, 3.

79. Oros. V, 6, 1-2: Seruio Fuluio Flacco Q. Calpurnio Pisone consulibus Romae puer ex ancilla natus quadripes quadrimanus, oculis quattuor, auribus totidem, natura uirili duplex. In Sicilia mons Aetna uastos ignes eructavit ac fudit, qui torrentum modo per prona praecipites proxima quaeque corripientibus exussere flammis, longinquiora autem favillis calidis cum uapore graui late uolitantibus torruerunt... In Bononiensi agro fruges in arboribus enatae sunt ; sul passo vd. anche BERTI, 1989 : 89-90 con ulteriori indicazioni bibliografiche.

80. Si veda Jul. Obs. 25 relativo all'anno 136 a. C. per l'attestazione della nascita di un neonato deforme e Jul. Obs. 26 relativo all'anno 135 a. C. a proposito dell'eruzione straordinaria dell'Etna e del grano spuntato sugli alberi nell'area di Bologna.

81. Oros. V, 6, 3: Igitur in Sicilia bellum seruile ortum est, quod adeo graue et atrox multitudine seruorum, instructu copiarum, magnitudine uirium fuit, ut, non dicam praetores Romanos, quos penitus profligavit, sed consules quoque terruerit.

82. Oros. V, 6, 4-6.

83. Oros. $\mathrm{V}, 11,1-5$.

84. Si legga in Jul. Obs. 30 relativo all'anno 125 a. C. il riscontro sulla comparsa in territorio africano di locustae che scagliate in mare e poi sospinte verso Cirene, avrebbero provocato con esalazioni e fetore intollerabili una strage di bestie e persone, nonché la precisa notizia sulle ottocentomila vittime morte a causa dell'epidemia.

85. Come lascia intendere la notizia che si legge in Liv. Per. LX, 4 ; su tale genere di eventi vd. anche Liv. XXX, 2, 10 ; XLII, 2, 5 ; XLII, 10, 7 ; Plin. Nat. XI, 105, nonché FOWLER, 1904 ; FEVRIER, $2003: 45$

86. Sulla scorta dell'ipotesi di LIPPOLD, 1976, II : 419 si veda Aug. De ciu. III, 31.

87. Oros. V. 11, 6 : Verumtamen pace et gratia omnipotentis Dei dixerim, de cuius misericordia et in cuius fiducia haec loquor: quamuis et temporibus nostris exoriantur aliquando et hoc diuersis partibus lucustae et plerumque etiam sed tolerabiliter laedant, numquam tamen temporibus Christianis tanta vis inextricabilis mali accidit, ut pernicies lucustarum, quae nullo modo ferri uiua potuisset, mortua plus noceret et qua diu uiuente peritura erant omnia, ea perdita pereuntibus magis omnibus optandum fuerit, ne perisset.

88. Per qualche approfondimento vd. SALvo, 2008.

89. Oros. V, 13, 3 : Eodem tempore Aetna mons ultra solitum exarsit et torrentibus igneis superfusis lateque circumfluentibus Catinam urbem finesque eius oppressit ita ut tecta aedium calidis cineribus praeusta et praegrauata conruerent: cuius leuandae cladis causa senatus decem annorum uectigalia Catinensibus remisit. 
90. Jul. Obs. 32.

91. Secondo cui Orosio trasse probabilmente ispirazione di nuovo da Aug. De ciu. III, 31 ; sul punto vd. anche BERTI, 1989 : 97-100.

92. Oros. V, 15, 20-21.

93. Oros. V, 15, 22 ; sull'episodio vd. anche Liv. Per. LXIII, 4 ; Cass. Dio XXVI, frg. 87 ; Plut. Quaest. Rom. 83, Ascon. Mil. 32 ; nonché LORSCH WILDFANG, $2006: 86$; SCARDIGLI, $2008:$ 204-205.

94. Jul. Obs. 37 : L. Heluius eques Romanus... Responsum infamiam uirginibus... Tres uno tempore uirgines Vestales nobilissimae... poenas subierunt.

95. Oros. V, 15, 19: Qui in triumpho ante currum cum duobus filiis suis actus et mox in carcere strangulatus est.

96. Si legga la celebre espressione attribuita a Giugurta in Oros. V, 15, 5 : quam cum egrederetur infami satis notauit elogio dicens: "o urbem uenalem et mature perituram, si emptorem inuenerit".

97. Oros. V, 18, 3-6 ; sulla selezione di eventi prodigiosi proposta nel passo cf. HAUG, 1947 : 111 ss \& 206-211.

98. Secondo cui Orosio avrebbe maldestramente sintetizzato le testimonianze relative ad un evento sismico accaduto nel 91 a. C. in agro Mutinensi ricordato da Plin. II, 199, nonché da Strab. VI, 1, 6 (qualora, presupponendo un errore per il toponimo Rhegion attestato nel testo, si identifichi la località ivi citata con Reggio Emilia e non Reggio Calabria) ; Jul. Obs. 54 ; sulla reale natura del fenomeno e sulla sua localizzazione vd. anche CASOLI, 2001:64-65; 68 per cui esso sarebbe stato in effetti avvistato su una strada di collegamento fra la Pianura Padana e la Toscana, non sulla via Emilia.

99. Cic. De diu. I, 99 da cui si ricava che prodigia e ostenta che avrebbero preceduto la guerra sociale erano stati registrati nelle Historiae di Sisenna.

100. Jul. Obs. 54 che attesta una sequela di prodigi correlabili con quelli menzionati da Orosio, ponendoli peraltro in connessione temporale con lo scoppio del bellum Italicum conseguente alle leggi emanate da Druso.

101. Oros. V, 18, 7 : Drusus tantis malis anxius domi suae incerto quidem auctore interfectus est.

102. Indicativa l'apertura del racconto (Oros. V, 18, 1-2) su quanto accaduto nel 91 a. C. : Anno ab urbe condita DCLVIIII Sex. Iulio Caesare et L. Marcio Philippo consulibus intestinis causis sociale bellum tota commouit Italia. Siquidem Liuius Drusus, tribunus plebi, Latinos omnes spe libertatis inlectos cum placito explere non posset, in arma excitauit.

103. Oros. V, 18, 8-10.

104. Oros. VI, 14, 4-5.

105. Oros. VII, 2, 11.

106. Jul. Obs. 65 .

107. Oros. VII, 39, 15.

108. Oros. VI, 20, 5-6.

109. Oros. VI, 20, 5-6: quasi eum unum ac potissimum in hoc mundo solumque clarissimum in orbe monstraret, cuius tempore uenturus esset, qui ipsum solem solus mundumque totum et fecisset et regeret... Quo signo quid euidentius quam in diebus Caesaris toto Orbe regnantis futura Christi natiuitas declarata est? Christus enim lingua gentis eius, in qua et ex qua natus est, unctus interpretatur.

110. Oros. VI, 20, 7 : hoc est per omne Romani tempus imperii, Christum et ex eo Christianos, id est unctum atque ex eo unctos, de meritoria taberna, hoc est de hospita largaque Ecclesia, affluenter atque incessabiliter processuros...

111. Sul legame di Orosio con la storiografia classica hanno insistito fra gli altri ZECCHINI, 1993 :

232 ; BRANDT, 2009.

112. Aug. De ciu. III, 31.

113. Oros. IV, 5, 10-13.

114. Per tale giudizio si veda Momigliano, 1975 : 98. 


\section{RIASSUNTI}

Guidato dal proposito di ripercorrere la storia umana con il fine di dimostrare la soggezione dell'umanità a mali d'ogni genere già nella fase anteriore all'affermazione del Cristianesimo, nelle Historiae adversus Paganos Orosio raccoglie notizie di eventi eccezionali, spesso calamitosi, come epidemie, terremoti, inondazioni, nascite mostruose, apparizioni improvvise di animali. L'esame dei passi dell'opera in cui tali episodi sono attestati relativamente all'età repubblicana e la comparazione con altre fonti (per es. Livio, Giulio Ossequente) consente di notare la tendenza di Orosio a porre talora in relazione eventi catastrofici distinti, in modo da rendere più cupo il quadro complessivo e la sua inclinazione a proporne una "riscrittura" comunque utile a far emergere con tono di critica e di condanna l'inefficacia delle pratiche pagane di volta in volta adottate o introdotte per attutirne gli effetti o placare gli dei, all'insegna di una prospettiva che talvolta appare analoga a quella manifestata da Agostino.

Visant à reparcourir l'histoire humaine comme preuve de l'asservissement de l'humanité aux maux de toutes sortes avant l'affirmation du Christianisme, dans ses Histoires contre les Païens Orose recueille les récits d'événements exceptionnels souvent calamiteux tels épidémies, tremblements de terre, inondations, naissances monstrueuses, apparitions imprévues d'animaux. L'examen des passages de l'œuvre où ces épisodes sont attestés pour l'âge républicain et la comparaison avec d'autres sources (par exemple Tite-Live et Julius Obsequens) permet de vérifier la tendance d'Orose à relier des événements catastrophiques distincts afin d'assombrir le tableau et sa préférence pour une « réécriture » capable de faire émerger sa critique de l'inefficacité des pratiques païennes introduites ou adoptées au cas par cas pour en atténuer les effets et apaiser les dieux, dans une perspective qui apparaît parfois semblable à celle d'Augustin.

\section{INDICE}

Mots-clés : Orose, histoire de la Rome républicaine, calamités, épidémies événements prodigieux

\section{AUTORE}

\section{IDA GILDA MASTROROSA}

Professore associato di « Storia romana », insegna all'Università di Firenze. Ha dedicato le proprie ricerche ad aspetti istituzionali e culturali della storia e della società romana, alla loro interpretazione nella tradizione antiquaria e ricezione nella cultura moderna. È autrice di studi riguardanti fra l'altro la storiografia romana di età imperiale (Livio, Tacito, Cassio Dione, Ammiano Marcellino), le strutture familiari e la condizione femminile in Roma antica, i riflessi della prassi giudiziaria e della normativa giurisprudenziale nella trattatistica retorica latina, nelle raccolte declamatorie e nell'epistolografia di età imperiale, l'elaborazione della propaganda 
politica in ambito romano.

idagilda.mastrorosa[at]unifi.it 